\title{
Event Servers for Crisis Management
}

\author{
K. Mani Chandy \\ California Institute of Technology \\ mani@cs.caltech.edu
}

\begin{abstract}
Organizations need to respond rapidly to threats and opportunities in the extended environment. Examples of threats are terrorism, chemical spills, intrusion into networks, and delayed arrivals of components. Opportunities include arbitrage and control of distributed systems such as the electrical power grid and logistics networks. The increasing prevalence of sensors such as RFID (radio frequency ID) and Web sites that offer measured information, coupled with information in databases and applications allows organizations to sense critical conditions in the extended environment. A response to a critical condition is to send an alert to appropriate people or send a message that initiates an application. A critical condition is a predicate on the history of global states of a system. An event server is a software component with sensors, responders, and computational elements. Computations are incremental in the sense that computations incorporate each new event in an online algorithm as opposed to repeatedly computing predicates over the entire history. This talk discusses the problem space, proposes an abstract mathematical model of the problem, suggests approaches to solutions, describes an implementation, and relates this work to research in data stream management systems and rules engines.
\end{abstract}

Biography: Mani Chandy received his B.Tech from IIT Madras in 1965 and his PhD from MIT in 1969 in Electrical Engineering. He worked in industry and taught at the University of Texas at Austin and is now at the California Institute of Technology. He has published papers in computer performance modeling, distributed systems, program correctness and event systems. He received the IEEE Koji Kobayashi Award and the CMG Michelson Award for contributions to computer performance modeling, and he is a member of the US National Academy of Engineering. 